\title{
Integrating Multiple Smart Directional Antennas for Full Potential Exploitation of Broadcasting System
}

\author{
R. Kalaiprasath ${ }^{1}$, R. Elankavi ${ }^{2}$, R. Udayakumar ${ }^{3}$ \\ ${ }^{1}$ Research Scholar, Department of CSE, BIHER, Bharath University, Chennai-600073, India \\ Assistant Professor, Department of CSE, Aksheyaa College of Engineering \\ ${ }^{2}$ Research Scholar, Department of CSE, BIHER, Bharath University, Chennai-600073 \\ ${ }^{3}$ Associate Professor, Department of Information Technology, Bharath University, Chennai, India
}

\begin{abstract}
In the new era of wireless telecommunication, the network consists of a broadcast server with a set of clients. It sends a group of information to the clients in a desired closed loop path. According to the information send by the broadcasting server the clients access it this should be happen in a cyclic path. In olden days we use fixed directional antennas for transmitting the signal from one place to another. Due to some drawback over the existing one we use multiple directional antennas at the Broadcast Server has been shown to increase performance. In many cases however, such broadcasting systems fail to exploit the full potential of the multiple antennas as they do not take into account the geographical distribution of clients within the coverage area of the system. This letter proposes an adaptive smart antenna [4] based wireless push system where the beam width of each smart antenna is altered based on the current placement of clients within the system area. Coupled with a modification of the broadcast schedule, the proposed approach significantly increases the performance observed by the system clients.
\end{abstract}

Keywords: MIMO, MISO, Broadcasting-Server, Multiple-Access Channel, Channel-State Information

\section{Introduction}

Wireless telecommunications refers to the transfer of information between two or more points that are not physically connected. Distances can be short, such as a few meters for television remote control, or as far as thousands or even millions of kilometers for deep-space radio communications. It encompasses various types of fixed, mobile, and portable applications, including two-way radios, cellular telephones, personal digital assistants (PDAs), and wireless networking.

Telecommunication is the science and practice of transmitting information by electromagnetic means. Communication is talking to someone or thing not necessarily through technological means. Telecommunication, however, is talking through technology meaning phones, Internet, radio etc...

In earlier times, telecommunications involved the use of visual signals, such as beacons, smoke signals, semaphore telegraphs, signal flags, and optical heliographs, or audio messages such as coded drumbeats, lung-blown horns, and loud whistles.

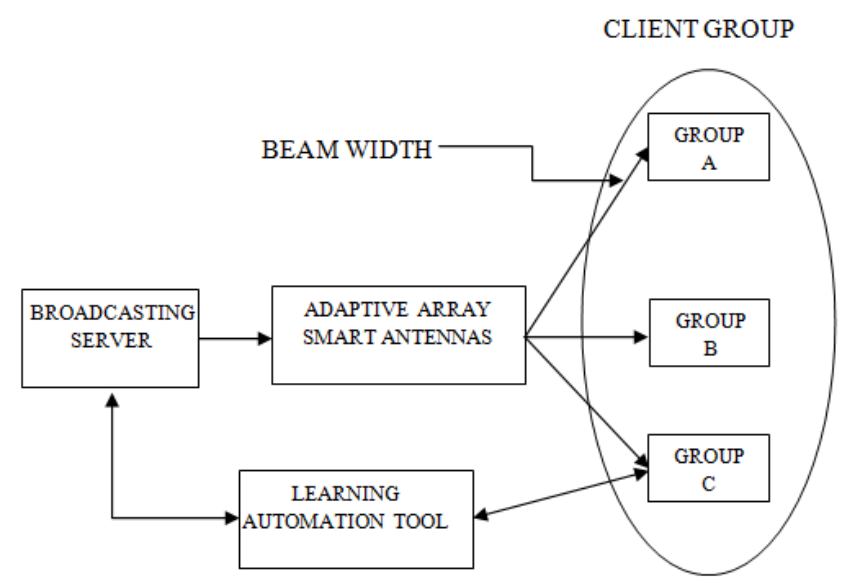

Figure 1: Block Diagram of the System

In modern times, telecommunications involves the use of electrical devices such as the telegraph, telephone, and teleprinter, as well as the use of radio and microwave communications, as well as fiber optics and their associated electronics, plus the use of the orbiting satellites and the Internet.

Data broadcasting is the broadcasting of data over a wide area via radio waves. It most often refers to supplemental information sent by television stations along with digital television, but May also be applied to digital signals on analog TV or radio. It generally does not apply to data which is inherent to the medium, such as PSIP data which defines virtual channels for DTV or direct broadcast satellite systems; or to things like cable modem or satellite modem, which use a completely separate channel for data. 


\section{International Journal of Science and Research (IJSR) \\ ISSN (Online): 2319-7064}

Index Copernicus Value (2013): 6.14 | Impact Factor (2014): 5.611

\subsection{Related Works}

The Directional antennas are used in communication systems for transferring information to the clients according to their needs. The yage-uda antenna and dipole antenna are some of the antennas used for communication purpose. In the existing system uses the directional antennas with fixed beam width.

The main drawback of this kind of antennas are fail to exploit the full potential of the multiple antennas as they do not take into account the geographical distribution of clients within the coverage area of the system, and also we cannot alter the beam width according to the client's need.

Due to the fixed beam width in directional antennas the some of the antennas handle more number of clients and some of them handle less number of clients this makes the distribution among the clients not-uniform, and also we cannot fix a set of clients to it.

\subsection{Contribution}

Due to some disadvantage over the existing system we propose another technique called smart antennas with rescheduling application. The use of multiple directional antennas at the Broadcast Server has been shown to increase performance.

This letter proposes an adaptive smart antenna based wireless push system where the beam width of each smart antenna is altered based on the current placement of clients within the system area. Coupled with a modification of the broadcast schedule, this should be done by using learning automaton tool on the broadcasting server side. The proposed approach significantly increases the performance observed by the system clients.

\subsection{System Technique}

\section{Learning Automaton [5]}

A learning automaton is an adaptive decision-making unit situated in a random environment that learns the optimal action through repeated interactions with its environment. The actions are chosen according to a specific probability distribution which is updated based on the environment response the automaton obtains by performing a particular action.

Learning Automation (LA) whose probability distribution vectors determines the popularity information item among the clients in service area of the antenna. The figure that represents the operation of the Learning automation is given below. By using this technique the system can find the popularity information, and it excludes the items that are never demanded by the clients in the coverage area of the antenna.

\section{Beam Forming [1]}

Beamforming can be used for radio or sound waves. It has found numerous applications in radar, sonar, seismology, wireless communications, radio astronomy, acoustics, and biomedicine. Adaptive beamforming is used to detect and estimate the signal-of-interest at the output of a sensor array by means of optimal spatial filtering and interference rejection. Beam forming is a signal processing technique used in sensor arrays for directional signal transmission or reception. Beam forming can be used at both the transmitting and receiving ends in order to achieve spatial selectivity.

Beam forming techniques are mainly used to change the directionality of the array. When transmitting, a beamformer controls the phase and relative amplitude of the signal at each transmitter, in order to create a pattern of constructive and destructive interference in the wave front.

Beamforming techniques can be broadly divided into two categories

1) Conventional (fixed or switched beam) beamformers

2) Adaptive beamformers or phased array

Conventional beamformers use a fixed set of weightings and time-delays (or phasinges) to combine the signals from the sensors in the array, primarily using only information about the location of the sensors in space and the wave directions of interest. In contrast, adaptive beamforming techniques generally combine this information with properties of the signals actually received by the array, typically to improve rejection of unwanted signals from other directions.

All the weights of the antenna elements can have equal magnitudes. The beamformer is steered to a specified direction only by selecting appropriate phases for each antenna. If the noise is uncorrelated and there are no directional interferences, the signal-to-noise ratio of a beamformer is given by $S N R=\frac{1}{\sigma_{N}^{2}} . P$

Where $\mathrm{P}=$ Transmitting power, $\sigma_{N}^{2}=$ Noise Power

\section{Wireless Push System and Using Smart Antennas}

\subsection{Wireless Push System}

In this literature we are also discussing about the mobile clients. The clients who are present in the broadcasting server should be a fixed or a mobile user. For the case of mobile user the users change their position for a particular time. So that we can find the corresponding position of the client so after that we have to transmit the signals. So for that we can find the location of the clients by using the below method.

The control message to obtain the clients location needs to be sent by the BS for each service area at the beginning of the system operation. Then this procedure will be repeated in a periodic manner after a fixed number of item broadcasts so as to update the client location information at the BS and use this information for rearranging the antenna beam widths in case the distribution of clients within the coverage area of the system differs from the previously estimated one.

\section{Volume 5 Issue 1, January 2016}




\section{International Journal of Science and Research (IJSR) \\ ISSN (Online): 2319-7064}

Index Copernicus Value (2013): 6.14 | Impact Factor (2014): 5.611

The absolute frequency of this procedure depends on the moving rate of the clients, and can be set at a small value when clients move at small speeds. However as will be seen from the simulation results in the next Section, the performance of the smart-antenna based push system is still significantly improved compared to that of a multiple antenna system of fixed beamwidth antennas even in cases where there exist users that move at higher speeds than others.

Using the procedure described above, apart from the efficient allocation of clients to the number of available antennas, each antenna is also set to exclude from its broadcast schedule the information items that refer to geographic areas that are out of its coverage. In this way no bandwidth is wasted to futile broadcasts, a fact that contributes to performance increase.

\subsection{Using Smart Antennas}

The multiple directional antenna system does not fully exploit the potential of the available directional antennas at the BS. This is attributed to the fixed way that these serve the coverage area due to their lack of ability for beamwidth alteration. Therefore a significant room for improvement exists in cases where some of the antennas cover areas with a high density of groups (thus they serve the majority of clients) while the other antennas cover areas with few or no groups.

To this end, the proposed system is equipped with smart antennas instead of directional ones. Based on their capability of altering their beamwidth, the use of smart antennas aims at allocating a similar number of clients to each antenna and thus to achieve a more efficient coverage of the broadcast area in cases where the distribution of clients within the system area is not uniform.

After that the information at the broadcasting server should be send as a control packet at each service location area. This aims to trigger $t$ trigger the group of clients at this location area to send back a feedback. This should be done mainly based on the coordinates of the coverage area contained on the control packet and its local coordinates that are available via its GPS receiver, each client will determine whether it needs to respond to the control packet with a feed back. Specifically, each such control packet transmits the actual coordinate sets that define the boundaries of the selected service location area. Thus, the clients that respond to a control packet via a feedback are the ones residing inside the boundaries of the corresponding selected service area and these clients are classified as a group to the BS.

After the above procedure is completed for all service locations, the system will obtain an estimate of both the total number of clients of the system and number of clients within each service area of the system.

By using the smart antenna we can also estimate the total number of clients present in for a particular antenna. By calculating this we can reduce the antenna overloading, by assigning the remaining clients to other smart antennas present in the system.

\section{Proposed Network Model}

Proposed network model is comprised of

1. Systems Characteristics And The Broadcasting Algorithm

2. Probability Updating Scheme

3. Performance Evaluation

\subsection{Systems Characteristics and the Broadcasting Algorithm}

In this module we have to design the basic system that consists of one broadcasting server and $\mathrm{N}$ number of clients. According to the population the clients are divided into several numbers of groups. Broadcasting server uses multiple antennas for transmitting the signals to the clients. According to the number of clients the antennas used on the broadcasting server should be changed.

Basic system consists of a broadcasting server and a group of clients. According to the number of clients antennas used at the broadcasting server should be changed. In this system we have to use smart antenna for the transmission of information to the clients. The main use of these kinds of antennas is they accept signal from all direction and also they adjust their beamwidth according to the client's location. It should be more advantage over the existing system.

We introduce an technique called Learning Automaton tool. This tool is mainly used to find the client requirement. Because the system used here is push in nature. So the clients want to demand their requirement to the broadcasting server. This should be carried out by using these types of tools at the BS.

The information sent from the BS to clients as a control packet, each information present in the broadcasting server should be arranged in a specific format according to their characteristics, they are said to be "Broadcasting Schedule". After the information sent by the broadcasting server it should be accessed by the group of clients, according to their response the broadcasting schedule should be arranged by using the learning automaton tool present in this system.

In the multiple antenna wireless push system each antenna is equipped with a LA that contains the server's estimate $p_{i}$ of the demand probability $d_{i}$ for each data item $i$ among the set of the items the antenna broadcasts.

$$
\sum_{i=1}^{N} p_{i}=\sum_{i=1}^{N} d_{i}=1
$$

Where $N$ is the number of items in the server's database.

The server estimates the next transmission by using the cost function present in this system. The cost function mainly used to find the next transmission, by comparing the current transmission with the previous transmission. 


\section{International Journal of Science and Research (IJSR) \\ ISSN (Online): 2319-7064}

Index Copernicus Value (2013): 6.14 | Impact Factor (2014): 5.611

$$
\mathrm{G}(\mathrm{i})=(\mathrm{T}-\mathrm{R}(\mathrm{i}))^{2} \mathrm{p}_{\mathrm{i} / \mathrm{li}}\left(\left(1+\mathrm{E}\left(\mathrm{l}_{\mathrm{i}}\right) / 1-\mathrm{E}\left(\mathrm{l}_{\mathrm{i}}\right)\right)\right.
$$

Where,$f^{\text {ee }}$ is the length of the item should be broadcast by the server. The length of the item should be calculated by using the equation (3).

$$
l_{i}=\operatorname{round}\left(\left(\frac{L_{1}-L_{0}}{M-1}\right)(i-1)+L_{o}\right), 1 \leq i \leq M
$$

Where L1 and Lo are the parameters are used to characterize the distributions,,$\dot{y}$ ' is the number of items present the system. Round () function used to give the rounded integer value at the output. the mean access time of the entire system for both fixed and smart antennas are given below in equation (4).

$$
T_{\text {opt }}=\frac{1}{2}\left(\sum_{i=1}^{M} \sqrt{p_{i} l_{i}}\left(\frac{1+E\left(l_{i}\right)}{1-E\left(l_{i}\right)}\right)^{1 / 2}\right)^{2}
$$

Where $\mathrm{E}(\mathrm{li})$ is the length of the item that are received erroneously by the clients and they are given by,

$$
E\left(l_{i}\right)=1-e^{-\lambda l_{i}}
$$

\subsection{Probability Updating Scheme}

Learning automata are mechanisms that can be applied to learn the characteristics of a system's environment. A learning automaton is an automaton that improves its performance by interacting with the random environment in which it operates. Its goal is to find among a set of $M$ actions the optimal one, so that the average penalty received by the environment is minimized. This means that there exists a feedback mechanism that notifies the automaton about the environment's response to a specific action. The operation of a learning automaton constitutes a sequence of cycles that eventually lead to minimization of average penalty. The learning automaton uses a vector, $P(n)=\left\{p_{1}(n), p_{2}(n), \ldots \ldots \ldots, p_{M}(n)\right\} \quad$ which represents the probability distribution for choosing one of the actions $a_{1}, a_{2}, \ldots ., a_{M}$ at cycle.

$$
\sum_{i=1}^{M} p_{i}(n)=1
$$

The core of the operation of the learning automaton is the probability updating algorithm, also known as the reinforcement scheme, which uses the environmental response triggered $\beta(n)$ by the action $a_{i}$ selected at cycle , $\mathrm{n}^{\text {ee }}$ to update the probability distribution vector , $\mathrm{p}^{\text {ec }}$. After the updating is finished, the automaton selects the action to perform at cycle $n+1$, according to the updated probability distribution vector $p(n+1)$.

$$
\mathrm{p}_{\mathrm{z}, \mathrm{j}}(\mathrm{K}+1)=\mathrm{p}_{\mathrm{z}, \mathrm{j}}(\mathrm{k})-\mathrm{L}\left(1-\beta_{\mathrm{z}}(\mathrm{k})\right)\left(\mathrm{p}_{\mathrm{z}, \mathrm{j}}(\mathrm{k})-\mathrm{a}\right), \forall \mathrm{j} \neq \mathrm{i}
$$

Where

$$
p_{z, i}(k) \in(a, 1), \forall i \in[1 . . N], L, a \in(0 \ldots 1), \quad \text { are }
$$

parameters of the LA. $L$ defines the rate of convergence, while the role of $\alpha$, is to prevent the probabilities of non- popular items from taking values very close to zero in order to increase the adaptivity of the LA.

Interference typically refers to the addition of unwanted signals to a useful signal. Common examples are:

- $\quad$ Electromagnetic interference (EMI)

- Co-channel interference (CCI), also called crosstalk

- Adjacent-channel interference (ACI)

- Intersymbol interference (ISI)

\section{Performance Evaluation}

In this module we make some performance calculation, system performance should be concluded by calculating the mean response time. Mean response time is the mean amount of time units that a client has to wait until it receives a desired information item. We consider $S A$ antennas having replicas of the same database of equally-sized items. The antennas are initially unaware of the demand for each item, so initially every item has the same probability estimate. Client demands are a-priori unknown to the server and location dependent. We consider $\mathrm{NumCl}$ clients that have no cache memory, an assumption also made in other similar research; Clients are grouped into $G$ groups each one located at a different geographical region. Any client belonging to group $g, 1 \leq g \leq G$, is interested in the same subset $S e c g$ of the server's database. All items outside this subset have a zero demand probability at the client. The items broadcast are subject to reception errors at the clients, with unrecoverable errors per instance of an item occurring according to a Poisson process with rate $\lambda$.

In this model we mainly calculate the system performance for both fixed and mobile users for various numbers of antennas. The system performance should be calculated by the mean response time of the group.

\subsection{System Characteristics and the Broadcasting Algorithm}

We are already discussing about the entire system and how we are develop that system that are already presented in the previous reviews. We are hereby introducing some more interesting topics for our presentation. The topology of the proposed wireless push system, an example of which is shown in below, consists of a large number of clients and a BS equipped with a number of smart antennas [2]. The fact that the system is of a push nature means that the system clients do not possess the ability to explicitly submit requests for data items, thus each client will wait for the item it demands to appear in the broadcast program constructed by the broadcast server.

In the proposed system, the ability of smart antennas to change their beam width is exploited so that the coverage area of each antenna is changed according to the current placement of clients within the system. This can be achieved by transmit beam forming, which allows a smart antenna to focus its transmit main beam towards the direction where the desired Client receivers reside and steer nulls in the other directions [4], so that clients residing in areas other 


\section{International Journal of Science and Research (IJSR) \\ ISSN (Online): 2319-7064}

Index Copernicus Value (2013): 6.14 | Impact Factor (2014): 5.611

than the desired one do not receive any transmission from this antenna. It has to be noted that such a requirement is nowadays easy to implement by already proposed smart antenna technology, which has gone even further by supporting Space Division Multiple Access (SDMA), a technique that requires from the smart antenna to form a transmission beam able to follow the movement of a specific mobile.

In this review we are mainly discussing about the various types of distributions and also discuss about the mobile users. In this paper we are mainly discussing about both fixed and mobile user. In previous reviews we are describing about the fixed user, so we move on to mobile users in this presentation.

\subsection{System Performance Increasing By Using Smart Antennas}

The multiple directional antenna system does not fully exploit the potential of the available directional antennas at the BS. This is attributed to the fixed way that these serve the coverage area due to their lack of ability for beamwidth alteration. Therefore a significant room for improvement exists in cases where some of the antennas cover areas with a high density of groups (thus they serve the majority of clients) while the other antennas cover areas with few or no groups.

To this end, the proposed system is equipped with smart antennas instead of directional ones. Based on their capability of altering their beamwidth, the use of smart antennas aims at allocating a similar number of clients to each antenna and thus to achieve a more efficient coverage of the broadcast area in cases where the distribution of clients within the system area is not uniform.After that the information at the broadcasting server should be send as a control packet at each service location area. This aims to trigger $t$ trigger the group of clients at this location area to send back a feedback. This should be done mainly based on the coordinates of the coverage area contained on the control packet and its local coordinates that are available via its GPS receiver, each client will determine whether it needs to respond to the control packet with a feed back. Specifically, each such control packet transmits the actual coordinate sets that define the boundaries of the selected service location area. Thus, the clients that respond to a control packet via a feedback are the ones residing inside the boundaries of the corresponding selected service area and these clients are classified as a group to the BS.

After the above procedure is completed for all service locations, the system will obtain an estimate of both the total number of clients of the system and number of clients within each service area of the system.

By using the smart antenna we can also estimate the total number of clients present in for a particular antenna. By calculating this we can reduce the antenna overloading, by assigning the remaining clients to other smart antennas present in the system.

\subsection{Wireless Push System for Mobile Clients}

In this literature we are also discussing about the mobile clients. The clients who are present in the broadcasting server should be a fixed or a mobile user. For the case of mobile user the users change their position for a particular time. So that we can find the corresponding position of the client so after that we have to transmit the signals. So for that we can find the location of the clients by using the below method [10].

The control message to obtain the clients location needs to be sent by the BS for each service area at the beginning of the system operation. Then this procedure will be repeated in a periodic manner after a fixed number of item broadcasts so as to update the client location information at the BS and use this information for rearranging the antenna beam widths in case the distribution of clients within the coverage area of the system differs from the previously estimated one. The absolute frequency of this procedure depends on the moving rate of the clients, and can be set at a small value when clients move at small speeds. However as will be seen from the simulation results in the next Section, the performance of the smart-antenna based push system is still significantly improved compared to that of a multiple antenna system of fixed beamwidth antennas even in cases where there exist users that move at higher speeds than others [9].Using the procedure described above, apart from the efficient allocation of clients to the number of available antennas, each antenna is also set to exclude from its broadcast schedule the information items that refer to geographic areas that are out of its coverage. In this way no bandwidth is wasted to futile broadcasts, a fact that contributes to performance increase.

\section{Simulation Results}

This section presents some simulation results about the system performance as show in fig. Here we are discussing about some graphical representation of the system performance. Mean response time for various numbers of fixed antennas is compared with the smart antennas. As compared to fixed antennas, smart antennas are providing uniform distribution among the group of clients.

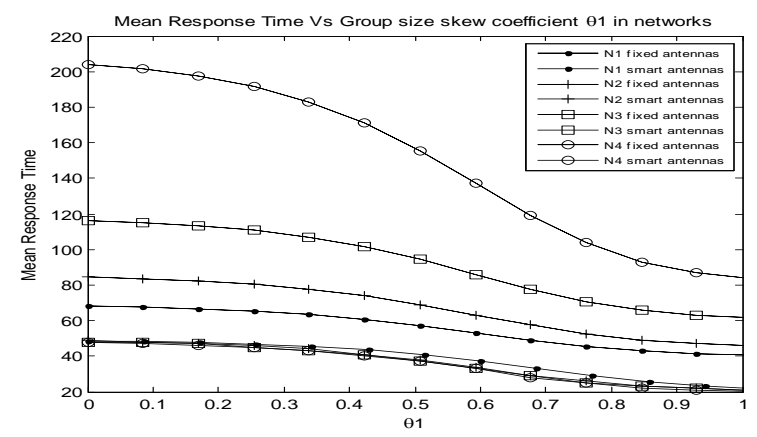

Figure 2: Fixed Antennas Vs Smart Antennas (Five Networks)

Mean response time for various numbers of fixed antennas is compared with the smart antennas. As compared to fixed antennas, smart antennas are providing uniform distribution among the group of clients.

\section{Volume 5 Issue 1, January 2016}




\section{International Journal of Science and Research (IJSR) \\ ISSN (Online): 2319-7064}

Index Copernicus Value (2013): 6.14 | Impact Factor (2014): 5.611

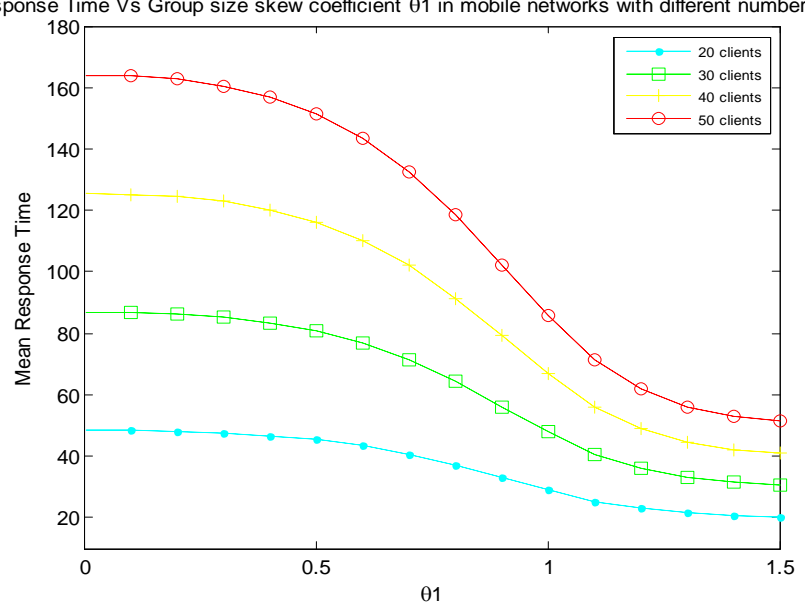

Figure 3: Response Time and the group size skew coefficient

This is the graph in between the Response Time and the group size skew coefficient. It shows that when the group size increases then the responding time for the Directional Smart Antennas decreases. For less number of clients this decrement in Response Time is less but for larger number of clients it becomes very high.The Mean Response Time is very less for the Smart Directional Antennas as shown in the graph.

\section{Conclusion}

This letter proposed an adaptive smart antenna-based wireless push system where the beamwidth of each smart antenna is altered based on the current placement of clients within the system. After the antenna assignment procedure, each antenna excludes from its broadcast schedule the information items that refer to geographic areas that are out of its coverage. Simulation results reveal that the abovementioned properties of the proposed system provide a significant performance increase over the system of that utilizes multiple antennas of fixed beam width.

\section{Acknowledgments}

I thank Spectrum Solutions, Pondicherry to help me for creating this paper with their Sincere Guidance and Technical Expertise in the field of communication.

I thank my guide Mr. K. Karthikeyan, A.P, PRIST University for his great support. The help of Mr.I.D.Soubache, HOD, Department of Electrical and Electronics PRIST University is really immense and once again I thank him for his great motivation.

I thank PRIST University, Pondicherry to provide me such a standard educational environment so that I am able to understand the minute concepts in the field of Engineering and Technology.

\section{References}

[1] Raushan Kumar Singh "Interference Cancellation and Rate Maximization in a Cognitive Networks",IJREAT, Volume 1, Issue 1, March, 2013
[2] C. Liaskos, S. Petridou, G "On the analytical performance optimization of wireless data broadcasting," IEEE Trans. Veh. Technol., vol. 59, no. 2, pp. 884-895, Feb. 2010.

[3] P. Nicopolitidis, A. S. Pompotsis, "Using learning automata for adaptive push-based data broadcasting in asymmetric wireless environments," IEEE Trans. Veh. Technol., vol. 51, no. 6, pp.1652-1660, Nov. 2002.

[4] Lal Chand Godara, "Smart Antennas", CRC Press ,Jan 2004.

[5] G. I. Papadimitriou, "Hierarchical discretized pursuit nonlinear learning automata with rapid convergence and high accuracy," IEEE Trans.Knowledge Data Eng., vol. 6, no. 4, pp. 654-659, Aug. 1994.

[6] A. Torkestani and M. Meybodi, "An efficient clusterbased

[7] CDMA/TDMA scheme for wireless mobile ad-hoc networks: a learning automata approach," J. Netw. Comput. Appl., vol. 33, no. 4, pp. 477-490, July 2010.

[8] J. ATorkestani and M. R. Meybodi, "An intelligent backbone formation algorithm for wireless ad-hoc networks based on distributed learning automata," Comput. Netw., vol. 54, no. 5, pp 826-843, Apr. 2010.

[9] P. Nicopolitidis, G. I. Papadimitriou, and A. S. Pomportsis, "Learningautomata- based polling protocols for wireless LANs," IEEE Trans. Commun., vol. 51, no. 3, pp. 453-463, Mar. 2003.

[10]K. Schreiner, "Where we at? Mobile phones bring GPS to the masses," IEEE Comput. Graphics Appl., vol. 27, no. 3, pp. 6-11, May-June 2007.

[11]N. H. Vaidya and S. Hameed, "Scheduling data broadcast in asymmetric communication environments," Wireless Netw., vol. 2, no. 3, pp. 171182, May 1999. 